\title{
A Linguistic Approach to Decisionmaking with Fuzzy Sets
}

\author{
RICHARD M. TONG AND PIERO P. BONISSONE, MEMBER, IEEE
}

\begin{abstract}
A technique for making linguistic decisions is presented. Fuzzy sets are assumed to be an appropriate way of dealing with uncertainty, and it is therefore concluded that decisions taken on the basis of such information must themselves be fuzzy. It is inappropriate then to present the decision in numerical form; a statement in natural language is much better. For brevity only a single-stage multiattribute decision problem is considered. Solutions to such problems are shown using ideas in linguistic approximation and truth qualification. An extensive example illuminates the basic ideas and techniques.
\end{abstract}

\section{INTRODUCTION}

$\mathrm{T}$ HE APPLICATION of fuzzy set theory to the problem of making a decision when only incomplete or uncertain information is available has been the subject of much research over the last decade (see Kickert [7] for a recent review). The basic premise behind this work is that there are situations where it is more natural to handle uncertainty by fuzzy set theory than by probability theory. While we agree with this, we do feel that most published work does not go far enough in its utilization of the fuzzy approach.

In this paper we present a technique for fuzzy decisionmaking that is based on linguistic approximation and truth qualification. The main feature, and advantage, of our approach is that it generates a linguistic assessment of the decision, thus making explicit the subjective nature of any choice that is made using fuzzy information.

In Section II of the paper we discuss multichoice decision problems in which information about the "suitability" of the alternatives is given by a set of fuzzy sets. We show how to generate a single fuzzy set that aggregates all the suitability information and how this may be interpreted as the basic fuzzy decision. Section III of the paper is concerned with the development of ideas in linguistic approximation and truth qualification. Following Zadeh [14], we introduce the concept of a truth-qualified proposition in natural language and show how this may be made the

Manuscript received January 7, 1980; revised July 8, 1980. This work was supported in part by a NATO Postdoctoral Research Fellowship from the Science Research Council of Great Britain (RMT) and also in part by Naval Electronic System Command Contract N00039-78-C-0013 (PPB).

R. M. Tong was with the Department of Electrical and Computer Sciences, University of California, Berkeley 94720 . He is now with Advanced Information and Decision Systems, 201 San Antonio Circle, Suite 286, Mountain View, CA 94040.

P. P. Bonissone is with the Corporate Research and Development Department, General Electric Company, P.O. Box 43, Building 37-579, Schenectady, NY 12301.

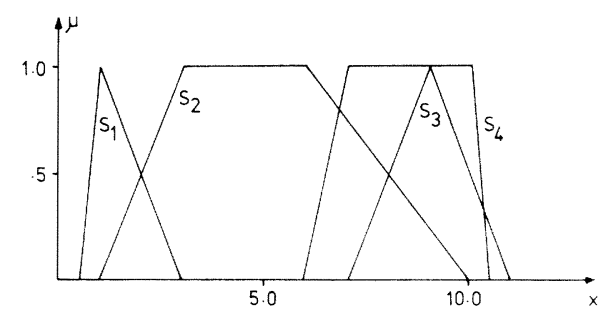

Fig. 1. Simple suitability sets.

basis for a linguistic decision. Finally, in Section IV we present a rather extensive example of an investment decision problem in which the ratings are linguistic rather than numerical. This example is designed both to illustrate our technique and to give further insight into the properties of the linguistic approach.

\section{A Multichoice Decision Problem}

We assume that the basic problem is to choose between a set of alternatives, $\mathbb{Q}=\left\{a_{i}: i=1, \cdots, m\right\}$, given some fuzzy information about the "suitability" of each of them. We also assume that this information is given as a set of fuzzy sets, $\delta=\left\{S_{i}: i=1, \cdots, M\right\}$, where each of the $S_{i}$ is defined by a membership function that maps the real line onto the closed interval $[0,1]$.

Suitability is simply interpreted as a measure of the ability of an alternative to meet our decision criteria. The concept arises quite naturally in this kind of problem and is essentially a fuzzification of the idea of a rating. Thus, for example, the $\delta$ could be fuzzy expected utilities in the manner of Watson [9], the fuzzy values described by Efstathiou and Rajkovic [5], or they might be computed from linguistic assessments of the alternatives with respect to the decision criteria. (We present an example of the latter in Section IV.)

Given this statement of the problem, we have to select the preferred alternative on the basis of $\mathcal{S}$ and then generate a linguistic statement about our decision. To help illustrate the selection procedure, consider the simple example of choosing between four alternatives given $S_{1}, S_{2}$, $S_{3}$, and $S_{4}$ as shown in Fig. 1.

It is ambiguous as to which alternative we should choose, but intuitively we would prefer either $a_{3}$ or $a_{4}$. To help us decide we shall introduce a concept of dominance that is closely related to Zadeh's [10] definition of separation. The separation $\sigma$ between two convex fuzzy sets $A$ 


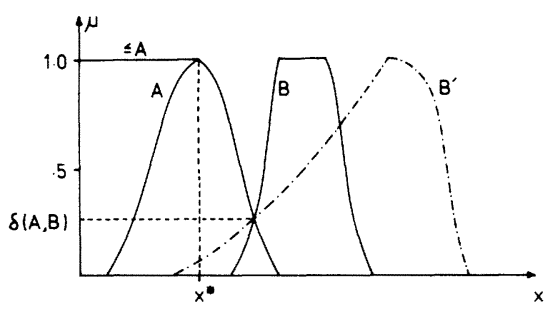

Fig. 2. Example of "less than or equal to A."

and $B$ is given by

$$
\sigma(A, B)=1-\bigvee_{x}\left(\mu_{A}(x) \wedge \mu_{B}(x)\right)
$$

where $\vee$ denotes the maximum operation and $\wedge$ denotes the minimum operation. We now define the dominance $\delta$ of $A$ over $B$ by

$$
\delta(A, B)=\underset{x}{\vee}\left(\mu_{<A}(x) \wedge \mu_{B}(x)\right)
$$

where $\leqslant A$ is the fuzzy set "less than or equal to $A$ " formed from $A$ by setting

$$
\begin{aligned}
\mu_{<A}(x) & =1.0, & & x<x^{*} \\
& =\mu_{A}(x), & & x \geqslant x^{*}
\end{aligned}
$$

with $x^{*}$ being the leftmost (i.e., lowest) value of $x$ for which $\mu_{A}(x)=1.0$. This is illustrated in Fig. 2. When the peak in $\mu_{A}$ is to the left of, or coincides with, the peak in $\mu_{B}$ we have $\delta(A, B)=1-\sigma(A, B)$. Notice that while $\delta(A, A)=1, \delta(A, B) \neq \delta(B, A)$ in general.

This definition is not the only one we could have chosen. Nonetheless, it does reflect our interest in the separation between the peaks of $\mu_{A}$ and $\mu_{B}$. Our basic reasoning is that the subset of $\delta$ which is nondominated gives a clear indication of the preferred alternatives. Since the universe of discourse on which the elements of $\delta$ are defined is a linearly ordered set, it follows that a nondominated fuzzy set is one with a peak which lies to the right of all the others. It is important to realize, however, that $\delta$ gives us no information about the overall shape of $A$ and $B$. Thus the set $B^{\prime}$ in Fig. 2 is such that $\delta(A, B)=$ $\delta\left(A, B^{\prime}\right)$. Clearly, then, we cannot rely solely on $\delta$ in forming our decision about $\mathbb{Q}$.

Our definition does allow us to construct a dominance relation $R_{\delta}$ on $\delta$. For the example of Fig. 1 this would be

$$
R_{\delta}=\left|\begin{array}{llll}
1.0 & 0.5 & 0.0 & 0.0 \\
1.0 & 1.0 & 0.8 & 0.5 \\
1.0 & 1.0 & 1.0 & 1.0 \\
1.0 & 1.0 & 1.0 & 1.0
\end{array}\right|
$$

where $R_{\delta}(i, j)$ is the dominance of $S_{i}$ over $S_{j}$. We see that there are two rows with all 1.0. Thus the subset of nondominated alternatives is $\left\{S_{3}, S_{4}\right\}$, and our intuition about the choice being between $a_{3}$ and $a_{4}$ is confirmed.

Although $R_{\delta}$ is reflexive, it is asymmetric and is generally nontransitive (see Zadeh [11] for a definition of these terms). It is not, therefore, a partial ordering on $\delta$. However, we can use it in generating a single fuzzy set which represents our decision. The procedure we adopt is very similar to one proposed by Baas and Kwakernaak [1].

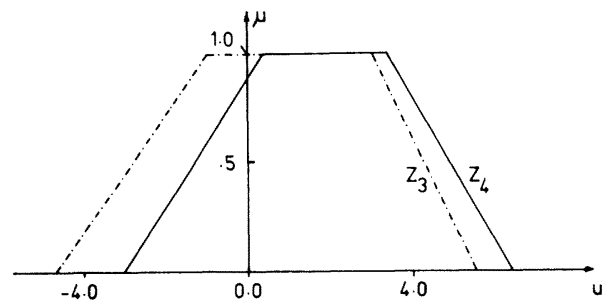

Fig. 3. Simple decision sets.

While their technique leads to an essentially numerical assessment of the choice, ours gives rise to a linguistic one.

First, we define a vector of weights $W$ such that $W(i)=\wedge R_{\delta}(i, j)$. These represent the overall degree to which one alternative dominates the others and may be thought of as defining a fuzzy set of dominant alternatives. Thus for the $R_{\delta}$ above $W=\left[\begin{array}{llll}0.0 & 0.5 & 1.0 & 1.0\end{array}\right]$.

As we have already remarked, this is not sufficient to make our choice since it takes no account of the shapes of the elements of $\mathcal{S}$. In order to do so, we define a difference function $g_{k}(\cdot)$ of $m$ independent variables,

$$
g_{k}\left(x_{1}, \cdots, x_{m}\right)=x_{k}-\frac{\sum_{\substack{i=1 \\ i \neq k}}^{m} W(i) \cdot x_{i}}{\sum_{\substack{i=1 \\ i \neq k}}^{m} W(i)}
$$

where the index $k$ corresponds to a position in $W$ for which $W(k)=1$. We can then compute the fuzzy preference set $Z_{k}$, induced by $g_{k}(\cdot)$ when $x_{i}$ is replaced by $S_{i}$. The "extension principle" (Zadeh [13]) defines this set to be

$$
\mu_{Z_{k}}(u)=\underset{\substack{\left(x_{1}, \cdots, x_{m}\right) \\ \text { s.t. } g_{k}(.)=u}}{\vee}\left[\bigwedge_{i=1}^{m} \mu_{S_{i}}\left(x_{i}\right)\right]
$$

which we write more compactly as $Z_{k}=g_{k}(\delta)$.

Note that Baas and Kwakernaak use a simpler difference function

$$
p_{k}\left(x_{1}, \cdots, x_{m}\right)=x_{k}-\frac{1}{m-1} \sum_{\substack{i=1 \\ i \neq k}}^{m} x_{i}
$$

which is clearly equal to $g_{k}(\cdot)$ if $W(i)=1.0$ for all $i$.

Our justification for $g_{k}$, and hence $Z_{k}$, is that when there are some clearly dominated alternatives these are relatively unimportant in coming to a final decision. In particular, if $W(i)=0$ for some $i$, then the corresponding alternative is definitely not preferred. The fuzzy set $Z_{k}$ is thus the fuzzy weighted difference between $S_{k}$ and $\left\{S_{i}: i \neq\right.$ $k\}$.

Since the weight vector for the example of Fig. 1 has two positions such that $W(k)=1$, that is $k=3$ and $k=4$, we have to form two preference sets, $Z_{3}=g_{3}(\delta)$ and $Z_{4}=g_{4}(\mathfrak{S})$, using our technique. These are shown in Fig. 3 and notice that our original assessment is reflected in the shapes with $Z_{4}$ being slightly to the right of $Z_{3}$. We might 
use this to assert that $a_{4}$ should be preferred to $a_{3}$ but our confidence in doing so should be rather low. Indeed, we would like a linguistic assessment of the situation, and we consider the problem of generating one in the next section.

\section{TRUTH QUalification AND Linguistic APPROXIMATION}

The central idea is that we shall express our preference for one of the alternatives over all the others as a truth qualified proposition in natural language. Thus our decisions will have forms similar to the following: "It is very true that $a_{k}$ is marginally preferred to all others," or "It is more or less true that $a_{k}$ is preferred to all others." These may be written more formally as " $a_{k}$ is $P$ to all others is $\tau$ " where $P$ is the strength of our preference and $\tau$ is the truth qualification.

Such decisions have three elements. First, there is the underlying nonfuzzy decision that picks out $a_{k}$. Second, there is the "strength" of this decision expressed as a linguistic degree of preference. Then third, there is some qualification of (or expression of confidence in) the decision in terms of the degree of truth. This three level format allows us considerable flexibility in forming decisions, and it gives a true sense of the uncertainty associated with the underlying data.

The problem of generating a linguistic decision from the preference set $Z_{k}$ is thus seen to be a matter of interpreting $Z_{k}$ in terms of our linguistic decision format. Essentially, all that is required is for us to give $Z_{k}$ a meaningful label. The method we use to do this is called linguistic approximation.

Before proceeding, we need to define what we mean by " $a_{k}$ is $P$ to all others is $\tau$." If we interpret $P$ as a fuzzy set on the same universe of discourse as $Z_{k}$ and if $\tau$ is a fuzzy truth set, then our decision statement can be translated into a fuzzy set with the same meaning. This concept of "semantic equivalence" was introduced by Zadeh [14] and gives an equivalent set, denoted $L$, defined by

$$
\mu_{L}(u)=\mu_{\tau}\left(\mu_{P}(u)\right) \text {. }
$$

Linguistic approximation then, is finding a fuzzy set $L$ which is close in meaning to $Z_{k}$.

To do this we utilize some recent work by Bonissone [2] on pattern recognition techniques applied to linguistic approximation. The first step is to generate a set of possible decision statements which we call the term set $E$. Each element of the set will be similar to the statements above although $P$ may be rather more complex as we shall see in the example.

The exact form of the term set will be problem dependent, but it should be large enough so that there is a wide range of possible decisions. To ensure this, the fuzzy sets $P$ and $\tau$ have to be chosen appropriately. Fig. 4 shows some typical preference sets which illustrate how $P$ may be defined. Because the universe of discourse is an ordered set, $P_{3}$ indicates a higher degree of preference to either $P_{1}$ or $P_{2}$. Similarly, $P_{2}$ has a higher degree than $P_{1}$. Thus we

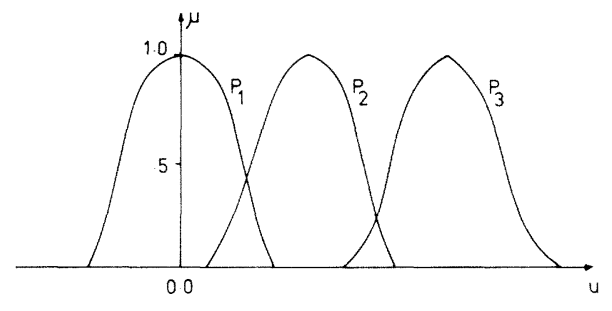

Fig. 4. Typical strength of preference sets.

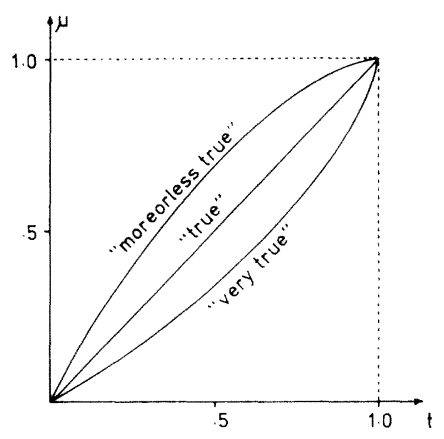

Fig. 5. Examples of truth sets.

can assign labels such as "indifferent," "marginally preferred," and "definitely preferred" to these sets.

Some fuzzy truth sets are shown in Fig. 5. Note especially that "true" is given by $\mu_{\text {"true" }}(t)=t$. This may seem counter-intuitive at first, but because of the way $\mu_{L}$ is defined, if $\tau$ is "true" then $L=P$. This gives the very reasonable result that a proposition of the form " $a_{k}$ is $P$ to all others is true" is the same as " $a_{k}$ is $P$ to all others."

Having fixed the primary $P$ and $\tau$, an efficient way of obtaining the term set is to use a context free grammar in the manner of Zadeh [12]. Thus if we consider each element of $\mathcal{L}$, denoted $L_{i}$, as a sentence in a synthetic language then all possible decision sentences can be generated using the grammar. Once this is done, we need to search among the elements of $\mathcal{E}$ for the one which is closest in meaning to the unlabeled $Z$. It is extremely inefficient to make pairwise membership function comparisons for all the $L_{i}$, so as a preliminary to performing a more efficient search we represent each of the $L_{i}$ by a pattern $p_{i}$ of characteristic features. Since the number of features is much smaller than the number of elements in the universe of discourse for $L_{i}$ (typically, four compared to 50) a search in this lower order space of features $\mathscr{P}$ can be performed much more effectively.

To do this we define a Euclidean metric in $\mathscr{P}$, denoted $d_{1}$, and find the set of $L_{i}$ which satisfy $d_{1}\left(p_{i}, p_{z}\right)<E$; where $p_{z}$ is the pattern for the unlabeled preference set and where $E$ is a parameter which defines our tolerance in judging the similarity of two patterns. The result of the search is thus a small subset of $\mathcal{L}$, denoted $L A[Z]$, which contains only those $L_{i}$ which are close in meaning to $Z$.

If we want a unique decision, as is usually the case, then we have to compare actual membership distributions. Thus we need to compare each $L_{i}$ in $L A[Z]$ with $Z$ using another appropriately defined metric. (In practice we have found a modified form of Bhattacharya distance to be 
especially effective.) If we denote this metric by $d_{2}$, then the linguistic decision statement corresponding to $Z$ is that $L_{i}$ in $L A[Z]$ which minimizes $d_{2}\left(L_{i}, Z\right)$. We denote this $L_{z}$ since it is the decision statement that labels $Z$. The reader is referred to Tong and Bonissone [8] for a detailed description of pattern space construction and the definitions of the metrics.

\section{An Investment Decision Problem}

As an illustration of the mechanics of our approach, let us consider the following problem. A private citizen has a moderately large amount of capital which he wishes to invest to his best advantage. He has selected five possible investment areas, $\left\{a_{1}, a_{2}, a_{3}, a_{4}, a_{5}\right\}$, and has four criteria, $\left\{c_{1}, c_{2}, c_{3}, c_{4}\right\}$, by which to judge them. These are

$a_{1}$ the commodity market.

$a_{2}$ the stock market.

$a_{3}$ gold and/or diamonds.

$a_{4}$ real estate.

$a_{5}$ long-term bonds.

and

$c_{1}$ the risk of losing the capital sum.

$c_{2}$ the vulnerability of the capital sum to modification by inflation.

$c_{3}$ the amount of interest received.

$c_{4}$ the cash realizability of the capital sum.

His rating of the alternatives with respect to the criteria, $\left\{r_{i j}: \mathrm{i}=1, \cdots, 5, j=1, \cdots, 4\right\}$, is expressed linguistically as shown in Table I. Thus, for example, his assessment is that there is a low risk of losing his capital if he invests in real estate and that the amount of interest received from stocks is only fair. His problem is to select one of the $a_{i}$ with the additional constraint that he does not consider the criteria to be equally important but gives them linguistic weights $\left\{\alpha_{1}, \alpha_{2}, \alpha_{3}, \alpha_{4}\right\}$, as shown in Table II. Thus the amount of interest received is the most important factor, whereas the cash realizability is the least important.

The first step in solving this problem is to compute a suitability set for each alternative. To do this we shall assume that a fuzzy weighted sum of ratings is the appropriate measure of suitability. Obviously, other models of this concept could be constructed for the problem, but our objective here is to show how to select an alternative given $\delta$ rather than to discuss the mechanism by which $\delta$ is obtained. So, we assume that

$$
S_{i}=\sum_{j=1}^{4} \alpha_{j} \cdot r_{i j}
$$

where the notions of "fuzzy addition" and "fuzzy multiplication" implied by this equation are derived by using the extension principle. Thus we translate the linguistic ratings and weights given in Tables I and II into fuzzy sets and then combine them using the equation above to give the suitability sets shown in Fig. 6.
TABLE I

LINGUISTIC RATINGS

\begin{tabular}{|c|c|c|c|c|c|}
\hline & \multicolumn{4}{|c|}{ Criteria } \\
\hline & & $c_{1}$ & $c_{2}$ & $c_{3}$ & $c_{4}$ \\
\hline & $a_{1}$ & high & $\begin{array}{c}\text { moreorless } \\
\text { high }\end{array}$ & $\begin{array}{l}\text { very } \\
\text { high }\end{array}$ & fair \\
\hline & $a_{2}$ & fair & fair & fair & $\begin{array}{l}\text { moreorless } \\
\text { good }\end{array}$ \\
\hline Alternatives & $a_{3}$ & low & $\begin{array}{c}\text { from fair } \\
\text { to } \\
\text { moreorless } \\
\text { low }\end{array}$ & fair & good \\
\hline & $a_{4}$ & Iow & $\begin{array}{l}\text { very } \\
\text { low }\end{array}$ & $\begin{array}{c}\text { moreorless } \\
\text { high }\end{array}$ & bad \\
\hline & $a_{5}$ & $\begin{array}{l}\text { very } \\
\text { low }\end{array}$ & high & $\begin{array}{c}\text { moreorless } \\
\text { low }\end{array}$ & $\begin{array}{l}\text { very } \\
\text { good }\end{array}$ \\
\hline
\end{tabular}

TABLE II

LINGUISTIC WEIGHTS

\begin{tabular}{|c|c|c|c|}
\multicolumn{1}{c}{$\alpha_{1}$} & $\alpha_{2}$ & $\alpha_{3}$ & $\alpha_{4}$ \\
\hline $\begin{array}{c}\text { moderately } \\
\text { important }\end{array}$ & $\begin{array}{c}\text { moreorless } \\
\text { important }\end{array}$ & $\begin{array}{c}\text { very } \\
\text { important }\end{array}$ & $\begin{array}{c}\text { moreorless } \\
\text { unimportant }\end{array}$ \\
\hline
\end{tabular}

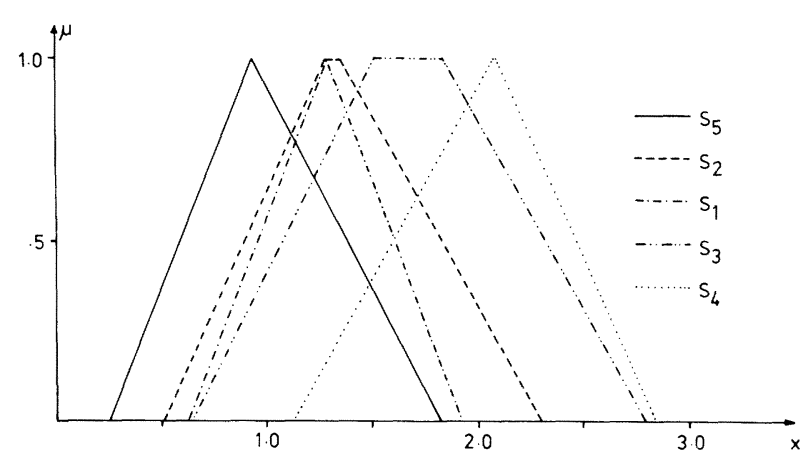

Fig. 6. Example, case 1 -suitability sets.

We have not included the definitions of $\alpha_{j}$ and $r_{i j}$ since these are not strictly relevant to our development. However, it is worth mentioning that provided they are restricted in certain ways, the actual computation of the $S_{i}$ can be considerably simplified. In particular, the work of Dubois and Prade [4] shows that when manipulating fuzzy sets it is often possible to work with parameterized versions of the membership functions rather than the membership functions themselves. In which case, applying the basic arithmetic operations between fuzzy sets becomes a matter of following some simple rules for combining the parameters.

The next step is to compute the dominance relation, which for our problem is

$$
R_{\delta}=\left|\begin{array}{lllll}
1.0 & 1.0 & 0.81 & 0.49 & 1.0 \\
1.0 & 1.0 & 0.90 & 0.58 & 1.0 \\
1.0 & 1.0 & 1.0 & 0.85 & 1.0 \\
1.0 & 1.0 & 1.0 & 1.0 & 1.0 \\
0.77 & 0.79 & 0.63 & 0.34 & 1.0
\end{array}\right|
$$

and we see that $S_{4}$ (real estate) dominates all the others, with the weights being [ $\left.\begin{array}{lllll}0.49 & 0.58 & 0.85 & 1.0 & 0.34\end{array}\right]$. We then apply the procedures described in the previous section first to compute $Z_{4}=g_{4}(\delta)$ and then to search for an appropriate linguistic approximation $L_{z}$. Note that we are 


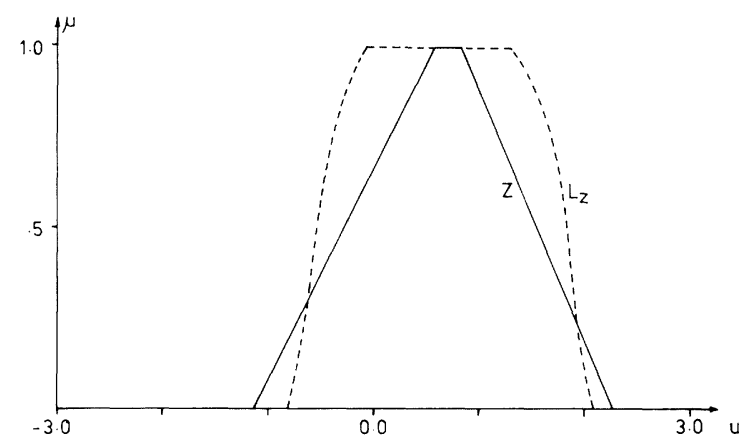

Fig. 7. Example, case $1-Z$ and $L_{z}$.

tacitly assuming the existence of a decision term set $\mathcal{E}$. In fact, of course, the generation of $E$ is an essential preliminary to this whole exercise. The membership functions for $Z_{4}$ and $L_{z}$ are as shown in Fig. 7.

Recalling that the standard form of decision is " $a_{k}$ is $P$ to all others is $\tau$," then we have that

$$
a_{k}=\text { "real estate" }
$$

and that $L_{z}$ is equivalent to

$P=$ "from indifferent with to marginally better than"

$\tau=$ "more or less true."

It is interesting to compare this linguistic statement with the dominance weights derived from $R_{\delta}$. In some sense, the $W(i)$ overemphasize the differences making it seem that real estate is a clear-cut choice. The linguistic statement, however, is rather more cautious. It picks out real estate, but makes us realize that the preference is only marginal.

This characteristic feature of the linguistic approach is, we feel, particularly valuable. In any decision problem where there is uncertainty in the data, there must be uncertainty in the decision itself. Obviously, one of the alternatives has to be selected, but we should be aware of the consequences of fuzzifying the problem.

Let us pursue this discussion by slightly modifying the problem. Suppose the investor changes his assessment of the importance of $c_{4}$. Instead of being simply "more or less unimportant" he feels it is "from more or less unimportant to moderately important." This gives a new set of suitability sets, see Fig. 8, and a new dominance relation,

$$
R_{\delta}=\left|\begin{array}{lllll}
1.0 & 1.0 & 0.91 & 0.58 & 1.0 \\
1.0 & 1.0 & 0.99 & 0.72 & 1.0 \\
1.0 & 1.0 & 1.0 & 1.0 & 1.0 \\
1.0 & 1.0 & 1.0 & 1.0 & 1.0 \\
1.0 & 0.96 & 0.81 & 0.51 & 1.0
\end{array}\right|
$$

The corresponding dominance weights are $\left[\begin{array}{lll}0.58 & 0.72 & 1.0\end{array}\right.$

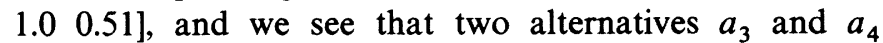
completely dominate the others. This means that we need to form two preference sets: one for real estate $Z_{4}$ and one for gold/diamonds $Z_{3}$. In fact these are so sufficiently similar that they have the same $L_{z}$, see Fig. 9 .

Thus our decision is that the investor is now indifferent

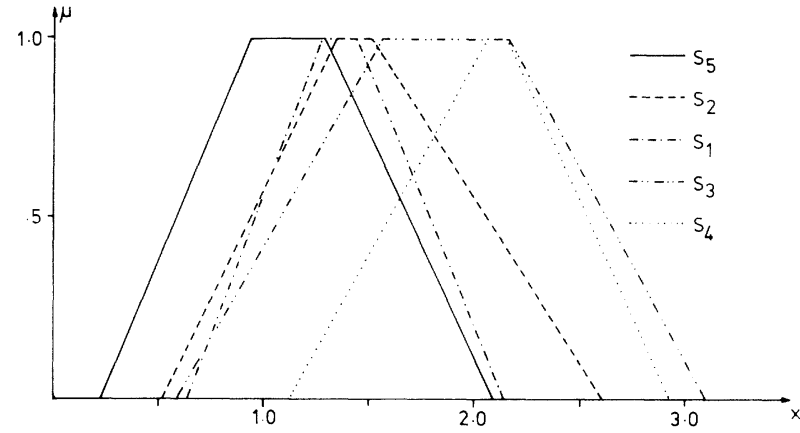

Fig. 8. Example, case 2-suitability sets.

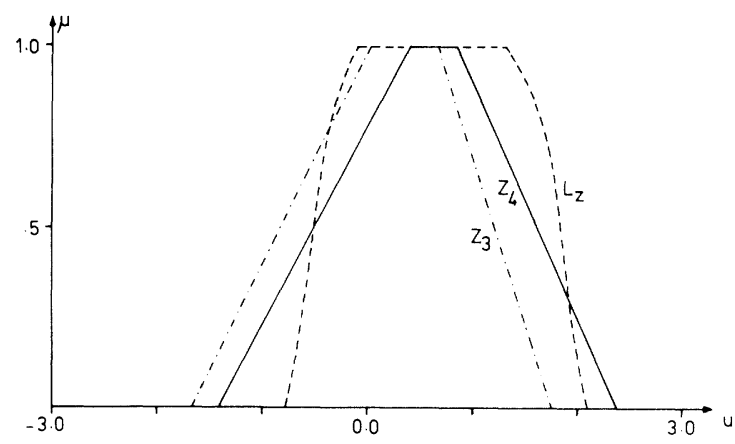

Fig. 9. Example, case $2-Z_{3}, Z_{4}$, and $L_{2}$.

to real estate and gold/diamonds and that $a_{k}=$ "both real estate and gold/diamonds"

$P=$ "from indifferent with to marginally better than"

$\tau=$ "more or less true."

This is a very interesting result. Inspection of Fig. 8 might have led us to believe that real estate would be preferred. However, we see that, once again, the linguistic approach emphasizes basic similarities rather than numerical differences.

If we wish to choose between real estate and gold/diamonds then we have to apply other criteria to $Z_{3}$ and $Z_{4}$ (or $S_{3}$ and $S_{4}$ ). We might think of selecting the one with least "fuzziness" or the one that gives the smallest possibility of low suitability. Alternatively, we might treat the problem as a binary choice decision and then reapply our method using just $S_{3}$ and $S_{4}$.

A better approach would be to assess the relative dominance of $a_{3}$ and $a_{4}$ with respect to the nondominant alternatives. By that we mean that two subproblems should be considered: the dominance of $a_{3}$ over $a_{1}, a_{2}$, and $a_{5}$, and the dominance of $a_{4}$ over $a_{1}, a_{2}$, and $a_{5}$. We form a dominance relation for each of these reduced problems by striking out the appropriate row and column in $R_{\delta}$. This gives

$$
\begin{aligned}
R_{R} & =\text { relative dominance of real estate } \\
& =\left|\begin{array}{llll}
1.0 & 1.0 & 0.58 & 1.0 \\
1.0 & 1.0 & 0.72 & 1.0 \\
1.0 & 1.0 & 1.0 & 1.0 \\
1.0 & 0.96 & 0.51 & 1.0
\end{array}\right|
\end{aligned}
$$


and

$$
R_{G}=\text { relative dominance of gold/diamonds }
$$

$$
=\left|\begin{array}{llll}
1.0 & 1.0 & 0.91 & 1.0 \\
1.0 & 1.0 & 0.99 & 1.0 \\
1.0 & 1.0 & 1.0 & 1.0 \\
1.0 & 0.96 & 0.81 & 1.0
\end{array}\right| .
$$

The corresponding dominance weights are

$$
\begin{aligned}
& W_{R}=\left[\begin{array}{llll}
0.58 & 0.72 & 1.0 & 0.51
\end{array}\right] \\
& W_{G}=\left[\begin{array}{llll}
0.91 & 0.99 & 1.0 & 0.81
\end{array}\right] .
\end{aligned}
$$

If we now define two fuzzy preferences

$$
Z_{R}=S_{4}-\left\{\frac{W_{R}(1) S_{1}+W_{R}(2) S_{2}+W_{R}(4) S_{5}}{W_{R}(1)+W_{R}(2)+W_{R}(4)}\right\}
$$

and

$$
Z_{G}=S_{3}-\left\{\frac{W_{G}(1) S_{1}+W_{G}(2) S_{2}+W_{G}(4) S_{5}}{W_{G}(1)+W_{G}(2)+W_{G}(4)}\right\}
$$

then we get a substantially different picture of the preference for real estate and gold/diamonds. Fig. 10 shows $Z_{R}$ with $L_{R}$ which corresponds to

$$
\begin{aligned}
a_{k}= & \text { "real estate." } \\
P= & \text { "between indifferent with and } \\
& \text { marginally better than." } \\
\tau & =\text { "more or less true." }
\end{aligned}
$$

and Fig. 11 shows $Z_{G}$ and $L_{G}$ which correspond to

$$
\begin{aligned}
a_{k} & =\text { "gold/diamonds." } \\
P & =\text { "between marginally worse than and marginally } \\
& \text { better than." } \\
\tau & =\text { "true." }
\end{aligned}
$$

Obviously, there is now a much stronger decision in favor of real estate.

Even though the difference is clear in this example, we should observe that, in general, this is not necessarily a straightforward problem. What is required is some way of actually ranking the dominant alternatives. Rather than ask whether "indifferent" plus "more or less true" is better than "marginally" plus "true," for example, it would be better to treat this final ranking as a separate problem. Thus, if we have determined that there is a dominant subset $\mathscr{D}$ of $\mathcal{S}$ such that these alternatives either have the same, or very similar $L_{z}$ then we should restrict our attention to only that subset. The problem is then to produce a rank ordering on $\mathscr{D}$.

As we have noted, previous attempts to solve the problem of ranking fuzzy sets are largely inadequate since they rely on numerical representations of the essential features of the problem and, therefore, defuzzify it. However, a more recent approach by Efstathiou and Tong [6] uses the concept of a linguistic preference relation and attempts to define linguistic equivalents of the classical ideas of transitivity and ordering. The essence of the approach is that the decisionmaker is required to make pairwise comparisons between the elements of $\mathscr{D}$ and to express his prefer-

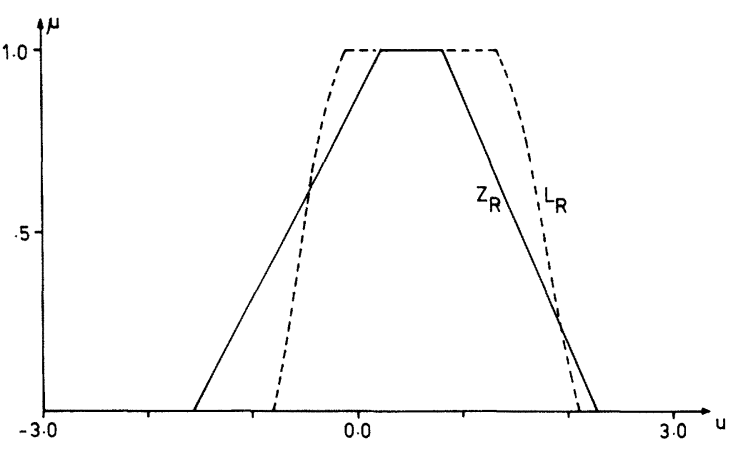

Fig. 10. Example, reduced problem $-Z_{R}$ and $L_{R}$.

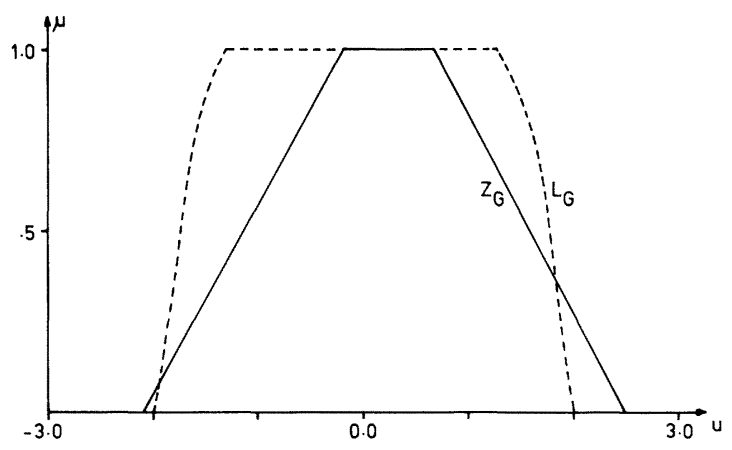

Fig. 11. Example, reduced problem $-Z_{G}$ and $L_{G}$.

ences linguistically. Analysis of the resulting preference relation allows a linguistic ordering to be constructed. While this work is only a preliminary study, it does show that such an approach is feasible and clearly preferable to any purely numerical method.

To summarize, it does seem that if there are two, or more, dominant alternatives, then we should form a decision for each of them without attempting to say which is the most dominant. We may well want to perform additional analysis on $\mathscr{D}$ so that we can pick just one alternative, but we should certainly not insist on calling this the "best" unless we have a very clear idea of what such a phrase means.

\section{Discussion}

We have focused on the theoretical aspects of linguistic decision analysis in the preceding sections, but it is important to realize that underlying these are some fundamental practical issues. To be of maximum value our technique has to be implemented as an interactive computer system. The prototype used to compute the example in Section IV is described in detail by Bonissone [3]. Essentially, the program flow follows the diagram shown in Fig. 12 and may be conveniently broken into two phases: a setting-up phase and a solution phase. In each phase the user interacts with the program by providing it with information about the problem. So, in the setting-up phase, all the parameters related to the problem formulation, decision set definition, and linguistic approximation 


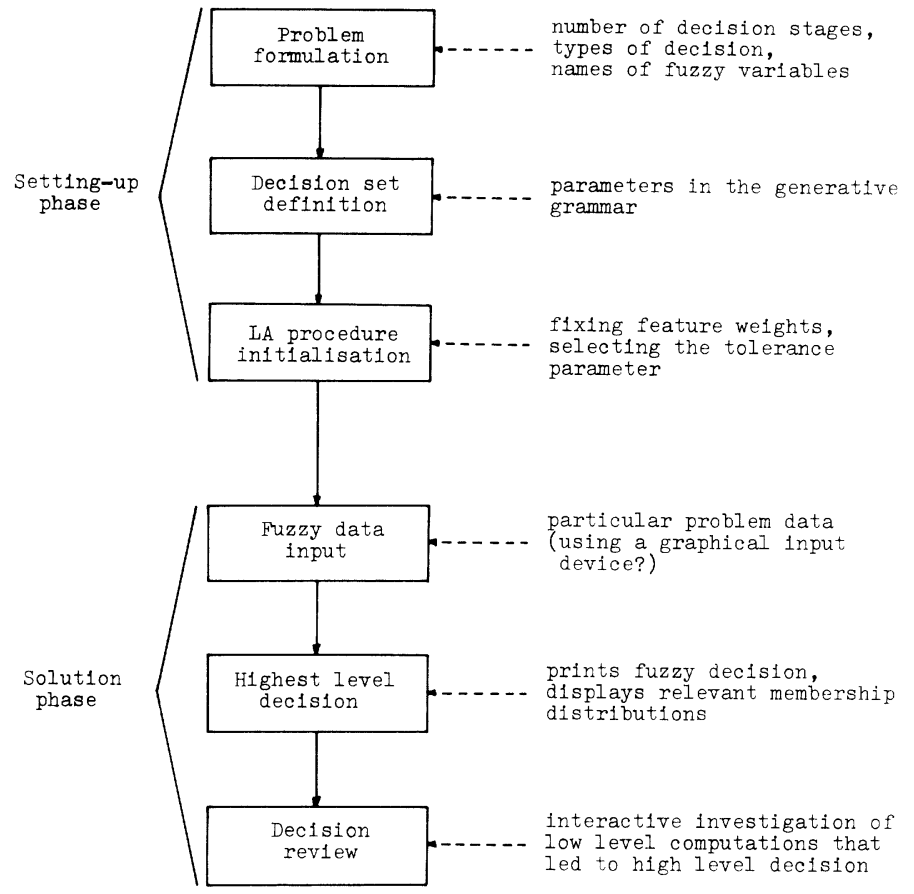

Fig. 12. Systems diagram for computer decision package.

initialization are defined. Then, in the solution phase, the decision algorithm is provided with the problem data, the solution is obtained, and its components are analyzed. Within this two phase structure we have indicated six distinct functions which are worth discussing in greater detail.

In the "problem formulation" step the actual problem under analysis is formally defined. The number of decision stages and their structure are specified and, for each decision stage, the number of alternative choices and the number of evaluating criteria are also determined. In our illustrative problem we have one decision stage, five alternatives, and four criteria.

In the "decision set definition" step we are concerned with the construction of the decision term set $\mathcal{L}$. This depends upon the primary terms we use for $P$ and $\tau$ as well as the actual parameters of the grammar. The user is expected to provide all this information, although some defaults may be acceptable in practice. In the illustrative example $P$ has seven primary linguistic values ranging from "definitely worse than" to "definitely better than," and $\tau$ also has seven primary values ranging from "very false" to "very true."

In the "linguistic approximation procedure initialization" step, all the parameters related to our linguistic approximation technique have to be entered. As in the previous step, it may well be possible to have default settings for most of these. In general, however, the user will have to provide information about the patterns of the characteristic features, the metrics $d_{1}$ and $d_{2}$, the tolerance parameter $E$, and several other parameters which are too technical for discussion here.

The "fuzzy data input" step uses information from the "problem formulation" step to set up an interactive dialogue with the user about his linguistic evaluations of the problem. At the end of this step, all information of the kind illustrated in Table I has been collected, and the solution to the problem can be computed using the procedures described in Section II.

In the "highest level decision" step, the decisionmaker is presented with the selected alternative $a_{k}$, the strength of the decision $P$, and the truth qualification $\tau$. Upon request, the system will also display the rank of the alternatives, the ranking values $W$, and a plot of the membership distributions of the preference set $Z$, and its approximation $L_{z}$.

In the "decision review" step another interactive dialogue can be initiated by the user to request information about the deep structure of the solution. Thus he can ask for the dominance relation $R_{\delta}$, the components of the suitability of the selected alternatives, the parameters of the linguistic approximation procedure, and other details. He may also plot the suitability sets for further insight into the problem.

If at this stage the decisionmaker is satisfied, then the problem is solved; if he is not, then he can reenter the program at any of the first four steps to change and modify the problem as he desires.

\section{Conclusion}

Previous attempts to solve fuzzy decision problems have produced numerical rankings of the alternatives. We believe that any attempt to do this is misguided. In situations where fuzzy sets are a suitable way of representing uncertainty, the decisions taken must be inherently fuzzy. It is surely not appropriate to give the final choice some artificial precision; decisions should be linguistic rather than numerical.

In developing a technique that generates linguistic decisions we have drawn heavily on the notions of linguistic approximation, pattern recognition, and fuzzy numbers. This has meant that our procedures are complex, though not complicated, and necessitate the use of a digital computer. However, the result is an easily used tool for structuring and solving fuzzy decision problems. The decisionmaker can interact with the decision process at every level and, as a consequence, we feel that our method is of direct practical benefit.

\section{ACKNOWLEDGMENT}

The authors are grateful for the use of the computing facilities provided by the Computer Centers of the University of California at Berkeley and at San Francisco. 


\section{REFERENCES}

[1] S. J. Baas and H. Kwakernaak, "Rating and ranking of multi-aspect alternatives using fuzzy sets," Automatica, vol. 13, pp. 47-58, 1977.

[2] P. P. Bonissone, "A pattern recognition approach to the problem of linguistic analysis in systems analysis," Proc. IEEE Int. Conf. Cybern. and Society, Denver, CO 1979.

[3] - "The problem of linguistic approximation in systems analysis," Ph.D. dissertation, Dep. EECS, Univ. California, Berkeley, 1979. (also Univ. Microfilms Int. Publ. \#80-14,618, Ann Arbor, MI).

[4] D. Dubois and H. Prade, "Operations on fuzzy numbers," Int. J. Syst. Sci., vol. 9, pp. 613-626, 1978.

[5] J. Efstathiou and V. Rajkovic, "Multiattribute decisionmaking using a fuzzy heuristic approach," IEEE Trans. Syst. Man, Cybern., vol. SMC-9, pp. 326-333, 1979.

[6] J. Efstathiou and R. M. Tong, "Ranking fuzzy sets using linguistic preference relations," Proc. 10th Int. Symp. Multiple-Valued Logic, Northwestern Univ., Evanston, IL, June 1980.

[7] W. J. M. Kickert, Fuzzy Theories on Decision Making: A Critical
Review, Leiden, Netherlands: Martinus Nijhoff, 1978.

[8] R. M. Tong and P. P. Bonissone, "Linguistic decision analysis using fuzzy sets," Electronics Research Lab., College of Engineering, Univ. California, Berkeley, Memo. UCB/ERL M79/72, 1979.

[9] S. R. Watson, J. J. Weiss, and M. L. Donell, "Fuzzy decision analysis," IEEE Trans. Syst. Man, Cybern., vol. SMC-9, pp. 1-9, Jan. 1979.

[10] L. A. Zadeh, "Fuzzy sets," Inform. Contr., vol. 8, pp. 338-353, 1965.

[11] - "Similarity relations and fuzzy orderings," Inform. Sci., vol. 3, pp. 177-200, 1971.

[12] - "Outline of a new approach to the analysis of complex systems and decision processes," IEEE Trans. Syst. Man, Cybern., vol. SMC-3, pp. 28-44, Jan. 1973.

[13] - "The concept of a linguistic variable and its application in approximate reasoning," Inform. Sci., vol. 8, pp. 199-249, 1975.

[14] - "PRUF - A meaning representation language for natural language," Int. J. Man-Machine Studies, vol. 10, pp. 395-460, 1978.

\title{
Aggregation and Information Structuring in Large-Scale Dynamic Systems
}

\author{
AMI ARBEL, MEMBER, IEEE, AND EDISON TSE, MEMBER, IEEE
}

\begin{abstract}
The problem of information structuring in large-scale dynamic systems is considered. The structuring effort is defined as the aggregation of the state vector in a prespecified manner. It is shown that, when the goal of the decisionmaking process (i.e., what are we trying to achieve) is considered in this aggregation step, simplified decision rules are obtained. This structured information is directly related to the goal of the decisionmaking process, and therefore, different goals will require different information structures even though the basic available data are the same; i.e., the same data have to be aggregated in a different way for every decision problem.
\end{abstract}

\section{INTRODUCTION}

$\mathrm{T}$ HE QUALITY of control inputs and decision strategies for dynamic systems is affected by the availability of information. The necessary information must be gathered, compressed, transmitted, and finally processed at the receiving station from which the control is issued.

Manuscript received February 11, 1980; revised July 17, 1980. This work was supported by the Office of Naval Research under ONR Contract N00014-75-C-0738.

A. Arbel was with Systems Control, Inc., Palo Alto, CA 94304. He is now with Advanced Information and Decisions Systems, 201 San Antonio Circle, Suite 286, Mountain View, CA 94040.

E. Tse is with the Department of Engineering-Economic Systems, Stanford University, Stanford, CA, 94305.
Cost and complexity is inherently associated with information delivery. The process is usually unstructured so that irrelevant information is mixed with relevant information. In large composite systems (i.e., systems comprised of interconnected subsystems), this information can be quite detailed and excessive. The use of such unstructured information slows down and complicates the decisionmaking process. This paper is concerned with developing information structures for the control of dynamic systems.

Structured information is the relevant, task-oriented, information to be specified by the decisionmaker. Relevant information depends on the task being performed.

The structuring of information involves specifying rules by which data are to be aggregated. Therefore, one can view the structuring effort as a preprocessing phase. It is important to point out, however, that different tasks will need different information structures, even if the basic available data are the same.

The motivation for developing and using structured information is to promote efficiency in the decisionmaking process. This is especially important for communication and control within a large system. 